\title{
Retinopatia diabética: estudo de uma associação de diabéticos
}

\author{
Diabetic retinopathy: study from a Diabetic Association
}

Sílvia Reg'ina Ramos ${ }^{(1)}$

Fábio Prado Sabbag' (2)

Daniela Busato ${ }^{(3)}$

André Basso Miranda ${ }^{(3)}$

Carlos Augusto Moreira Júnior ${ }^{(4)}$

\section{RESUMO}

Objetivo: Avaliar a prevalência de retinopatia diabética (RD) em uma associação de diabéticos, bem como o nível de informação dos pacientes a respeito desta doença.

Métodos: $O$ estudo avaliou, aleatoriamente, 46 pacientes de uma associação de diabéticos que responderam a perguntas sobre conhecimento da RD e avaliação oftalmoscópica prévia e foram submetidos à exame de fundo de olho por oftalmoscopia binocular indireta.

Resultados: Entre os diabéticos tipo 1, o estudo encontrou 44,44\% dos pacientes com RD e um caso de RD proliferativa; entre os diabéticos tipo $2,24,3 \%$ possuíam $\mathrm{RD}$; $65,22 \%$ dos indivíduos avaliados nunca haviam feito exame de oftalmoscopia, embora $80,43 \%$ já haviam tido alguma informação sobre RD.

Conclusões: Concluímos que há baixa cobertura em termos de prevenção e detecção da RD nesta associação de diabéticos, o que contrasta com o nível de informação dos pacientes sobre a doença e com o fato de ser uma entidade voltada exclusivamente para o tratamento do diabete.

Palavras-chave: Diabete melito; Retinopatia.

\section{INTRODUĈ̃̃̃O}

O diabete melito (DM) está entre as principais causas de cegueira irreversível no Brasil. A doença atinge 7,6\% da população, segundo o Ministério da Saúde ${ }^{1}$. O diabete melito insulino-dependente (DMID ou tipo 1) representa aproximadamente $20 \%$ dos casos de DM primário, sendo o restante diabete melito não insulino-dependente(DMNID ou tipo 2) ${ }^{2}$. De forma geral, as complicações oftalmológicas são de alta freqüência e severidade no paciente diabético. Cerca de $50 \%$ sofrem estas complicações ${ }^{3}$. A retinopatia é a complicação crônica mais comum do diabete ${ }^{1}$. A prevalência de RD varia conforme o tipo de diabete e o tempo de evolução da doença. Se considerarmos o mesmo tempo de evolução de 10 anos, ela está presente em cerca de $90 \%$ dos DMID e $65 \%$ dos DMNID ${ }^{4}$. Enquanto que a prevalência de doença proliferativa em 15 anos é muito menor em DM tipo 2, a presença de edema macular em função da duração da doença é igual nos 2 grupos ${ }^{5}$.

Os seguintes fatores têm sido relacionados com uma evolução mais desfavorável da doença retiniana: idade ${ }^{6}$ (pior na fase pré-puberal), duração da doença ${ }^{7}$, raça (pior na negra), sexo (mais severa em mulheres), hipertensão arterial sistêmica e controle glicêmico ${ }^{8}$.

$\mathrm{O}$ objetivo deste trabalho é avaliar a prevalência de $\mathrm{RD}$ e o nível de informação a respeito desta doença entre pacientes de uma associação de diabéticos, por tratar-se de um grupo voltado para o esclarecimento e prevenção. 


\section{MATERIAL E MÉTODOS}

Este trabalho foi realizado no Departamento de Oftalmologia do Hospital de Clínicas da Universidade Federal do Paraná.

Incluímos no estudo, aleatoriamente, 46 pacientes de uma associação de diabéticos, 15 do sexo masculino e 31 do feminino, tendo os mesmos sido divididos em tipo 1 e 2 de acordo com a idade à época do diagnóstico. O protocolo constituía-se de anamnese e exame oftalmológico. A anamnese incluía: idade, sexo, tipo de diabete, tempo de diagnóstico, doenças associadas, se o paciente já havia realizado exame prévio de fundo de olho e se este já tinha algum tipo de informação sobre retinopatia diabética. No exame oftalmológico constava a avaliação da acuidade visual, aferição da pressão intraocular através do tonômetro de aplanação e fundoscopia com oftalmoscópio binocular indireto. Este era realizado em todos os pacientes pelo mesmo examinador.

Adotamos a seguinte classificação para os estágios da RD ${ }^{9}$.

1. RD não proliferativa (RDNP):

- leve - ausência de edema de pólo posterior, microaneurismas e/ou microhemorragias em um quadrante.

- moderada - exsudatos duros e/ou manchas algodonosas, anormalidades microvasculares intra-retinianas (IRMA).

- grave - microhemorragias e microaneurismas nos quatro quadrantes.

2. RD proliferativa (RDP):

- presença de neovascularização, hemorragia vítrea ou descolamento de retina.

Quanto à metodologia estatística, recorreu-se à análise descritiva dos dados através de tabelas, quadros e gráficos. Para a comprovação do objetivo desse trabalho, foram utilizados os testes não-paramétricos "Qui-Quadrado com correção de Yates", "Exato de Fisher" (através do software "Epi-Info") e "Comparação entre duas Proporções" (obtido através do software "Primer of Biostatistics") para amostras independentes. O nível de significância (ou probabilidade de significância) mínimo adotado foi de $5 \%$.

\section{RESULTADOS}

As mulheres representaram $67,4 \%$ da amostra e os homens, $32,6 \%$. Entre os 46 pacientes, encontramos 13 casos de RD $(28,26 \%)$, sendo $6(46,15 \%)$ entre os homens e $7(53,85 \%)$ entre as mulheres. Do total de homens, $40 \%$ tinham RD e das mulheres, $22,6 \%$.

Nove diabéticos (19,56\%) eram portadores de DM tipo $1 \mathrm{e}$ 37 (80,44\%) de DM tipo 2. Dos diabéticos tipo 1, 44,44\% (4 pacientes) apresentaram RD e dos tipo 2, 24,3\% (9 pacientes).

Encontramos retinopatia proliferativa apenas em DMID; $50 \%$ dos casos de RD em DMNID eram do tipo não proliferativa grave e $50 \%$ não proliferativa leve e moderada (Tabela 1). O tempo médio de diagnóstico foi 13 anos para a

\begin{tabular}{|c|c|c|c|c|c|c|}
\hline \multirow{2}{*}{$\begin{array}{l}\text { Retinopatia } \\
\text { Diabética }\end{array}$} & \multicolumn{2}{|c|}{ Tipo I } & \multicolumn{2}{|c|}{ Tipo II } & \multicolumn{2}{|c|}{ Total } \\
\hline & $\mathbf{N}^{\circ}$ & $\%$ & $\mathbf{N}^{\circ}$ & $\%$ & $\mathbf{N}^{\circ}$ & $\%$ \\
\hline Não Proliferativa & 03 & 75,0 & 09 & 100,0 & 12 & 92,3 \\
\hline Proliferativa & 01 & 25,0 & - & - & 01 & 7,7 \\
\hline TOTAL & 04 & 100,0 & 09 & 100,0 & 13 & 100,0 \\
\hline
\end{tabular}

$\mathrm{RD}$ não proliferativa e 14 para a $\mathrm{RD}$ proliferativa; para os homens foi de 10,4 anos e para as mulheres 7 anos e para o diabete tipo 1 foi de 18,25 anos, enquanto que para o tipo 2 de 7,11 anos. O tempo de diagnóstico foi maior no diabete tipo 1, apesar de esta diferença não ter sido estatisticamente significativa $(\mathrm{p}=0,412)$.

Dos portadores de RD, $46,15 \%$ já haviam sido submetidos a pelo menos um exame de fundoscopia e dos não portadores de RD, 30,3\% já haviam feito o exame; $65,22 \%$ dos diabéticos avaliados nunca fizeram fundoscopia. Sobre o conhecimento da doença, $80,43 \%$ já haviam tido alguma informação sobre RD (Tabelas 2 e 3 ).

A doença mais comumente associada que encontramos foi hipertensão arterial sistêmica - HAS (26,08\%), seguida de cardiopatias $(8,69 \%)$ e hipercolesterolemia $(6,52 \%) ; 66,67 \%$ dos indivíduos com hipercolesterolemia, $25 \%$ dos cardiopatas e $16,67 \%$ dos hipertensos apresentaram RD.

\section{DISCUSSÃO}

Embora tenha sido encontrada uma proporção maior de $\mathrm{RD}$ entre os homens, este achado não foi estatisticamente significativo $(\mathrm{p}=0,379)$, o que condiz com a literatura, na qual não há diferença da prevalência de RD entre os sexos ${ }^{1}$.

\begin{tabular}{|c|c|c|c|c|c|c|}
\hline \multirow{2}{*}{$\begin{array}{l}\text { Fez fundo } \\
\text { de olho }\end{array}$} & \multicolumn{2}{|c|}{ Com RD } & \multicolumn{2}{|c|}{ Sem RD } & \multicolumn{2}{|c|}{ Total } \\
\hline & $\mathbf{N}^{\circ}$ & $\%$ & $\mathbf{N}^{\circ}$ & $\%$ & $\mathbf{N}^{\circ}$ & $\%$ \\
\hline Sim & 06 & 46,2 & 10 & 30,3 & 16 & 34,8 \\
\hline Não & 07 & 53,8 & 23 & 69,7 & 30 & 65,2 \\
\hline TOTAL & 13 & 100,0 & 33 & 100,0 & 46 & 100,0 \\
\hline
\end{tabular}

\begin{tabular}{|c|c|c|c|c|c|c|}
\hline \multirow{2}{*}{$\begin{array}{l}\text { Informação } \\
\text { sobre R.D. }\end{array}$} & \multicolumn{2}{|c|}{ Com RD } & \multicolumn{2}{|c|}{ Sem RD } & \multicolumn{2}{|r|}{ Total } \\
\hline & $\mathbf{N}^{\circ}$ & $\%$ & $\mathbf{N}^{\circ}$ & $\%$ & $\mathbf{N}^{\circ}$ & $\%$ \\
\hline Sim & 10 & 76,9 & 26 & 78,8 & 36 & 78,3 \\
\hline Não & 03 & 23,1 & 07 & 21,2 & 10 & 21,7 \\
\hline TOTAL & 13 & 100,0 & 33 & 100,0 & 46 & 100,0 \\
\hline
\end{tabular}


Houve maior prevalência $(\mathrm{p}<0,0001)$, assim como estágios mais avançados $(\mathrm{p}<0,005)$ de retinopatia nos diabéticos tipo 1, com resultados estatisticamente significativos.

Apesar da amostra relativamente pequena, que não representa todos os pacientes da associação, julgamos baixo o número de exames de fundo de olho realizados previamente, principalmente por tratar-se de pacientes de uma associação voltada para o conhecimento e tratamento de diabéticos. Considerando que nesta população de diabéticos, $65,22 \%$ nunca haviam realizado o exame e que entre os portadores de $\mathrm{RD}$, apenas $45,15 \%$ já haviam sido submetidos à fundoscopia, fica claro que estes números estão distantes do ideal para atingir uma prevenção adequada. Isto contrasta com o nível de informação que estes pacientes apresentaram $(80,43 \%$ já haviam tido informação sobre a RD), tendo pelo menos noção que o DM pode determinar perda visual irreversível. A literatura mostra que um screening para detecção precoce da RD pode salvar a visão por um custo relativamente baixo ${ }^{10}$.

O diabete é uma doença freqüentemente manejada por clínicos e cabe aos mesmos ou aos endocrinologistas a iniciativa da prevenção primária e secundária da RD. O ideal é que o exame de fundoscopia seja feito pelo oftalmologista, que realizaria o exame oftalmológico completo. Segundo a American Diabetes Association (ADA) este exame deveria ser realizado anualmente, nos casos de DMID, começando 3 a 5 anos após o diagnóstico mas não antes da puberdade e nos casos de DMNID iniciando logo após o diagnóstico ${ }^{4}$, pois este pode ser diagnosticado com um atraso de 5 a 7 anos e costuma se manifestar inicialmente por suas complicações, permanecendo por períodos variáveis de tempo de forma assintomática, com 30 a $50 \%$ dos doentes desconhecendo a própria enfermidade, ao contrário do DMID ${ }^{3}$.

A doença associada ao diabete mais encontrada foi a HAS, porém foram os pacientes com hipercolesterolemia os que apresentaram mais RD. Ambas são enfermidades freqüentemente associadas a diabete melito na literatura.

\section{CONCLUSÃO}

Verificamos o baixo nível de prevenção da retinopatia realizado pelos pacientes da associação estudada.

Acreditamos que a associação pode contribuir de forma mais adequada para prevenção e controle desta grave complicação do diabete melito.

\section{SUMMARY}

Purpose: To evaluate the prevalence of diabetic retinopathy $(D R)$ in a diabetic association, as well the level of knowledge about the entity among patients.

Methods: This study included 46 patients, at random, from a diabetic association that answered questions about DR and previous ophthalmoscopic examination. Then, they were submitted to indirect ophthalmoscopic evaluation.

Results: Among type 1 diabetic patients, the study found $44.44 \%$ of patients with DR and one case of proliferative retinopathy; among type 2, 24.3\% of pacients with DR; $65.22 \%$ of the total had never been submitted to ophthalmoscopic examination, although $80.43 \%$ had already received some information about $D R$.

Conclusions: This study shows that there is low coverage of preventive and detection examinations for $D R$ in this diabetic association, which contrasts with the acceptable level of the patients knowledge, besides being an association directed only to diabetic patients.

Keywords: Diabetes; Retinopathy.

\section{REFERÊNCIAS BIBLIOGRÁFICAS}

1. Diabete Melito. Guia Básico Para Diagnóstico e Tratamento. Ministério da Saúde do Brasil. Sociedade Brasileira de Diabete. Programa Harvard/ Joslin/ SBD, Brasília, 1996. pgs. 5, 7 e 57.

2. Foster D. Diabete Melito. In: Harrison. Medicina Interna. $13^{\circ}$ ed. GuanabaraKoogan, 1995;2:2079-101.

3. Consenso sobre prevenção, controle e tratamento do DMNID da ALAD (Associação Latinoamericana de Diabete), apresentado no $9^{\circ}$ Congresso Latinoamericano de Diabete, realizado em Foz do Iguaçu, 1996.

4. Zoorob RJ, Hagen MH. Guidelines on the Care of Diabetic Nephropathy, Retinopathy and Foot Disease. American Family Physician 1997;56(8):2021-8.

5. Diabetes Control and Complications Trial Research Group. The effect of intensive treatment of diabetes on the development and progression of longterm complications in insulin-dependent diabetes mellitus. N Engl J Med 1993;329:977-86.

6. The EURODIAB IDDM Complications Study. Retinopathy and Vision Loss in Insulin-dependent Diabetes in Europe. Ophthalmology 1997;104(2):252-60.

7. Vaughan D, Asbury T, Riordan-Eva P. In: Oftalmologia Geral. $4^{\circ}$ ed. Atheneu, 1998. p. 199-202.

8. Lima ALH, Ribeiro MBD, Belfort Jr. R. Prevalência de diferentes patologias e causas de cegueira em pacientes atendidos em serviço universitário de São Paulo. Arq Bras Oftal 1982;45:193-7.

9. Prior MJ et al. C-peptide and classification of diabetes melitus patients in the Early Treatment Diabetic Retinopathy Study. Report number 6. The EDTRS Research Group. Ann Epidemiol 1993;3(1):9-17.

10. Javitt JC, Aiello LP, Chiang Y, Ferris FL. 3d, Canner JK, Greenfield S. Preventive eye care in people with diabetes is cost-saving to the federal government. Implications for health-care reform. Diabetes Care 1994;17:409-17.

\section{Novidades na Internet:?!}

Agora no site CBO você tem disponível todas as informações na íntegra dos

Arquivos Brasileiros de Oftalmologia

httpe//www.cho.com.br/abo 